\title{
Osteoclast biology in bone resorption: a review
}

\author{
Chao FU ${ }^{1}$, Ruyi SHI ${ }^{2, *}$ \\ ${ }^{1}$ College of Fundamental Medicine, Shanxi Medical University, Taiyuan, Shanxi 030001, China. \\ ${ }^{2}$ Key Laboratory of Cellular Physiology, Chinese Ministry of Education; Department of Cell Biology and Genetics, Shanxi Medical University, Taiyuan, \\ Shanxi 030001, China. \\ *Correspondence: tomruyi@163.com \\ https://doi.org/10.37175/stemedicine.v1i4.57
}

\begin{abstract}
What we know about bone resorption has changed a lot in the last few decades. The osteoclast is the only cell to nibble and break down the bone, and in the formation and resorption of bone tissue, osteoclasts play an important role. Once the balance of bone formation and bone loss is out of control, diseases like osteopetrosis and osteoporosis occur. Bone resorption is a unique function of osteoblasts, which are multinucleated cells formed by the fusion of mononuclear progenitor cells of the monocyte/macrophage family. In the formation of osteoclasts, there are two main factors affecting this process, macrophage colony-stimulating factor (M-CSF) and ligand-activated receptor (RANKL) of nuclear factor kappa B (NF$\mathrm{KB}$ ). The identification of RANK-RANKL signaling and other classic signaling pathways such as Wnt and Notch, as the major signaling regulation in osteoclast differentiation, was a significant breakthrough in the field of osteoclastogenesis. In this review, we briefly describe the latest knowledge of osteoclast-induced bone resorption and cellular factors that regulate the activity of osteoclasts and cell fusion, for the purpose of understanding osteoclastogenesis and the development of drugs that enhance bone resorption to improve pathological bone diseases.
\end{abstract}

Keywords: Osteoclast $\cdot$ Bone resorption $\cdot$ M-CSF $\cdot$ RANKL pathway $\cdot$ Cell fusion

\section{Introduction}

Bone is the hardest organ in the body that makes up the internal bones of vertebrates. Its function is moving, supporting and protecting the body and storing minerals. One of the components of the bone is mineralized skeletal tissue with a solid honeycomb-like 3D structure inside, and there are tissues include bone marrow, periosteum, nerves, blood vessels and cartilage in the bone.

Osteoclasts are described as the only cells to be capable of destroying the bone tissue. They express markers that are thought to be specific to skeletal tissues, such as avb3 integrin, cathepsin K (CTSK), calcitonin receptor, and tartrate-resistant acid phosphatase (TRAP) (1). The osteoclast is essential for the two basic processes of bone biology, the first is bone modeling, which forms bone elements and ensures the correct shape and density of the bone. The second process is bone remodeling, and the mechanism of bone remodeling ensures bone tissue

Received: May 30, 2020; Accepted: Sep 3, 2020.

(c) The Author(s). 2020 This is an Open Access article distributed under the terms of the Creative Commons License (http://creativecommons.org/licenses/by/4.0/) which permits unrestricted use, distribution, and reproduction in any medium or format, provided the original work is properly cited. renewal and adapting to the environment. The balance of bone remodeling and resorption is essential for health. When the functions of osteoclasts and osteoblasts are disordered, it will cause bone diseases. The exacerbated bone resorption is associated with osteoporosis, rheumatoid arthritis and periodontal disease, while decreased bone resorption leads to osteopetrosis, a rare genetic disease. On the other hand, except for the function of bone resorption, osteoclasts are able to regulate cells in the bone marrow (2). We give this concise summary of previous work to better understand the osteoclast differentiation and identification of potential therapeutic targets (3).

\section{The differentiation of osteoclasts}

There are two basic groups in the blood cells which are differentiated by hematopoietic stem cells (HSCs): lymphoid lineage and myeloid lineage, such as macrophages. Osteoclasts are derived from the hematopoietic system (4). In the bone marrow, HSCs experience several differentiation and self-renewal, each subtype of the cell has a specific surface marker. There are four stages in the osteoclast differentiation: 1. HSCs differentiate into multipotential progenitor cells (c-Fms-, 


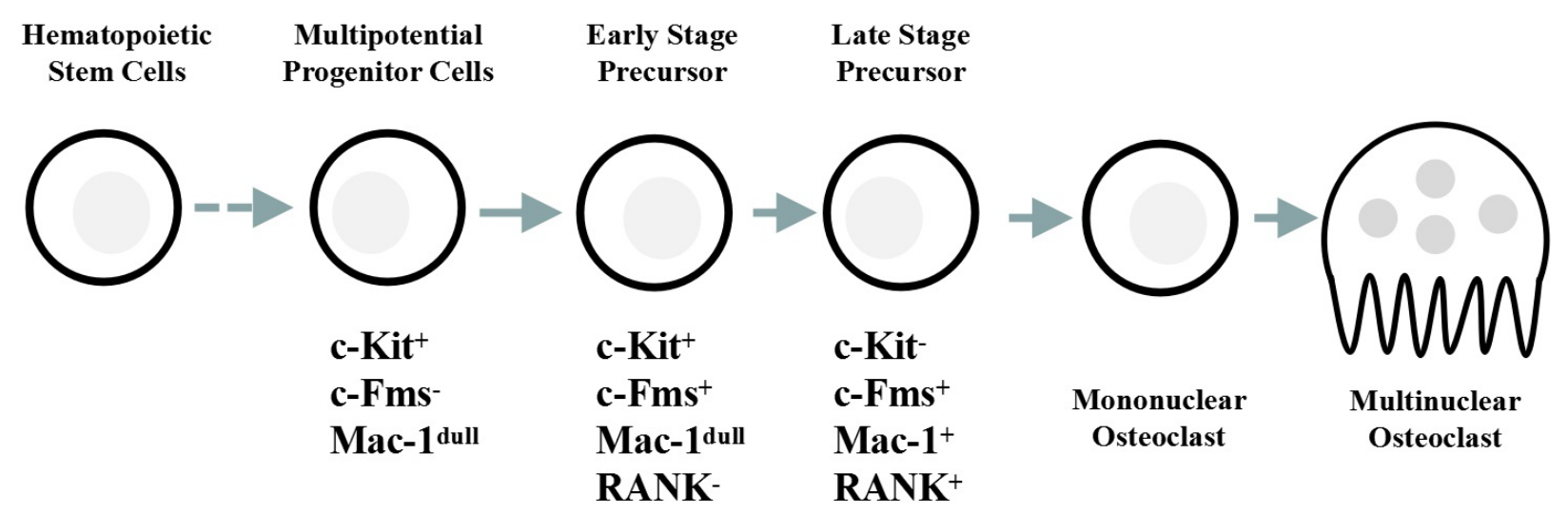

Figure 1. Stages in osteoclast differentiation. Phenotypic changes during the osteoclast differentiation and regulatory cytokines in the maturation and differentiation of osteoclasts.

c-Kit+, Mac-1dull); 2. multipotential progenitor cells differentiate into early-stage precursor (c-Fms+, c-Kit+, Mac-1dull, RANK-) and late-stage precursor (c-Fms+, c-Kit+, Mac-1+, RANK+); 3. precursors differentiate into mononuclear osteoclasts; 4. mononuclear osteoclasts differentiate into multinuclear osteoclasts (Figure 1) (5). D. G. Walker found that the capacity of resorbing bone was restored in osteopetrosis mice with intravenous administration of normal spleen and bone marrow cells (6). Interestingly, the dendritic cells are undergoing trans-differentiation into functional osteoclasts by stimulated with the microbe and the presence of receptor activator of nuclear factor kappa $B$ $(\mathrm{NF}-\kappa \mathrm{B})$ ligand (RANKL). Furthermore, The activation of dendritic cell-derived osteoclasts can be inhibited by aspirin which downregulates the expression of NFATc1 via the NF- $\kappa$ B pathway (7).

The differentiation of osteoclasts is mainly regulated by two critical cytokines, RANKL and macrophagecolony stimulating factor (M-CSF). PU.1 is a domain transcription factor of myeloid, B-lymphoid cells also regulate the transcription of $\mathrm{c}-\mathrm{fms}$ and $\mathrm{CD} 11 \mathrm{~b} / \mathrm{CD} 18$ which controls the osteoclast phenotype (8).

Mature osteoclasts are large whose size is up to $100 \mu \mathrm{m}$, multinucleated and polarized, firmly adhering to the surface of the bone. In the process of bone resorption, there are four different cell structures of osteoclast: 1. sealing zone, isolates the resorptive area from the extracellular environment; 2. ruffled board, facing the bone matrix, is composed of the plasma membrane to absorb the bone matrix; 3 . basolateral membrane, facing the vascular compartment, is participating in bone resorption, which contributes to transporting the bone degradation products; 4 . functional secreted domain.

\section{Factors and pathways which regulate the formation of osteoclasts}

M-CSF is critical for osteoclastogenesis (5). It is well known that the differentiation of osteoclasts needs two key molecules: NF- $\kappa$ B ligand RANKL and M-CSF (9). Osteoclasts are derived from the monocyte/macrophage cell line, and PU.1 controls the switch of activation of stem cell precursor. PU.1 drives a positive regulation of the M-CSF receptor, called c-fms. In the next step, the precursor becomes an osteoclast lineage by M-CSF and RANKL. On the other hand, macrophage or B lymphocyte can also trans-differentiate into an osteoclast. $\mathrm{C}$-fms supports the progenitors to survive and give rise to osteoclastogenesis by inducing the expression of RANK. Malt1-/- mice induced M-CSF production which played an important role in osteoclastogenesis and decreased osteoprotegerin (OPG) production while in the presence of inflammatory stimuli (10). GM-CSF can induce bone marrow-derived macrophages to differentiate to dendritic cells (11). M-CSF knockout mice (op/op mice) causes osteopetrosis by inducing the numbers of osteoclasts (12). RANKL is one member of the tumor necrosis factor superfamily and belongs to type II transmembrane protein. It is crucial for bone metabolism. It is a membrane protein (also known as CD254) on the osteoblast membrane that can activate the osteoclast and accelerate the formation of osteoclast and bone loss, which is important for bone regeneration. When RANKL on the osteoblast membrane activates the RANK protein on the osteoblast membrane, osteogenesis will begin (13). RANK is the critical receptor that mediates the function of osteoclasts on bone resorption and remodeling (14). Study shows that, when RAW264.7 type CRL-2278 cell line was cultured in the hydroxyapatite surface, the hydroxyapatite induces autocrine of RANKL and RANK by monocyte/macrophage cells to differentiate itself into osteoclasts (15). NF- $\mathrm{KB}$ is an essential factor for the differentiation of osteoclasts (16). The overexpression of OPG results in osteopetrosis by decreasing the differentiation of osteoclasts (Figure 2) (17). Several kinds of research have shown that the RANK/ RANKL/OPG pathway is a key signal pathway in bone metabolism and bone diseases resulting in imbalances in bone formation and resorption. RANK-/- mice (18), RANKL-/- mice (14), and the overexpression of OPG in rat (19) have shown symptoms of osteopetrosis. On the other hand, adolescent and adult OPG-/- mice developed osteoporosis and arterial calcification (20). The RANK/ RANKL/OPG pathway tightly and precisely controls the balance between bone formation and resorption. Some 
bone diseases in human patients such as familial expansile osteolysis (FEO) and the familial form of early-onset Paget's disease of bone (PDB2) occur when this process is out of control. In 2010, an investigation has found an extremely rare bone disease called dysosteosclerosis in a 3-year-old girl, the "Osteoclast-Poor" symptoms are similar to osteopetrosis caused by the deficiency of osteoclasts, but there are no mutations in the genes that encode RANK, RANKL, OPTG or M-CSF (21). How the lack of bone resorption occurs in this disease is still unclear.

Recent research has found that the salt-inducible kinase (SIK) signaling pathway takes part in the checkpoints of controlling the osteoclast formation. SIK inhibitors may denote a potential new treatment for preventing bone erosion (22). The SIKs are a serine/threonine kinase subfamily which belongs to the AMP-activated protein kinase (AMPK) family (23). SIK inhibitors can reduce the expression of osteoclast differentiation markers, such as TRAP and CTSK. Furthermore, the levels of c-Fos and NFATc1 proteins have an extreme downregulation when SIK inhibitors are present (22). $\mathrm{Pkn} 3$ bound to c-Src is critical for the activity of bone resorption by osteoclasts, while in the Wnt5a-Ror2 signal pathway, Pkn3 bound to c-Src can enhance the activityof osteoclasts (24). The Wnt signaling pathway has been extensively reported in osteoblasts lineage, but less known in osteoclasts. Recent studies found that early Wnt3a treatment inhibited the activation of NFATc1, which was also activated during macrophage differentiation into osteoclasts (25). Moreover, injection of microRNA-410 or downregulated Wnt-11 inhibited osteoclast function in osteonecrosis of the femoral head (26). On the other hand, the Notch signaling pathway plays a different role in osteoclastogenesis: in the osteoclast precursor cells, the stimulation of the Notch signal leads to large osteoclast with numerous nuclei and the activity of resorption but depresses the small osteoclast resorptive activity (27).

\section{Osteoclastic bone resorption}

The cytoskeleton of osteoclasts is unique because the polarization forms different sections to comply with different functions. The ruffled border is the isolated structure that shapes an actin-ring or sealing zone to segregate the absorption microenvironment from the outer space of the cell. This process needs the presence of $\alpha v \beta 3$ integrin. $\alpha v \beta 3$ integrin combined with the M-CSF receptor c-Fms to activate a signaling pathway constituted with Vav3, Syk, Dap12, c-Src, Slp76 and Rac which switches on the formation of actin-ring (28). $\beta 3$ gene knockout mice show that $\beta 3-/-$ cells are still multinucleated and express TRAP but are not able to form normal actin-ring, indicating osteoclast cytoskeleton dysfunction (29). In the contacting area between osteoclasts and bone surface, matured osteoclasts release enzymes like CTSK and TRAP to destroy the minerals. In healthy physical condition, there is high expression of TRAP by osteoclasts, activated macrophages and neurons (30). Under some pathological circumstances, TRAP expression is increased. These

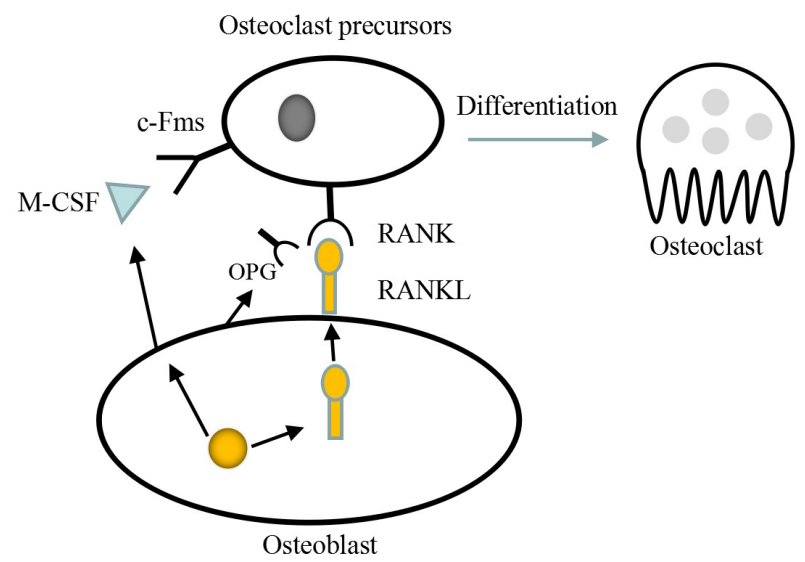

Figure 2. Regulation of osteoclast differentiation by osteoblasts through M-CSF and RANKL.RANKL and M-CSF expressed by osteoblasts are essential for osteoclast differentiation and fusion into multinucleated cells. On the other hand, OPG inhibits osteoclast differentiation by blocking the combination of RANKL and RANK.

diseases include osteoclastoma and osteoporosis, as well as metabolic bone disease. CTSK is expressed mainly in osteoclasts, which is a protease that performs the function bone resorption by catabolizing elastin, collagen and gelatin to break down bone tissues (Figure 3) (31).

Recently, some evidence has shown that the inhibitors of CTSK like 2-(3-(2-fluoro-4methoxyphenyl)-6-oxo-1(6H)pyridazinyl)-N-1H-indol-5-ylacetamide is a potential therapeutic drug for osteoporosis (32). Kent et al using the specific marker and time-lapse found that the resorption model of osteoclasts was not only the formation of a round pit, more importantly and more aggressively, there were long trenches that osteoclasts resorbed while moving (33).

To develop the capability of resorbing bone matrix, polarization is required. Through an area named sealing zone, osteoclasts can communicate with the bone and form an isolated zone with the bone matrix. This process requires the participation of molecules like c-Src, vitronectin receptor, carbonic anhydrase and CTSK (34). The osteoclasts secret protons through the ruffled border membrane to acidify the bone surface. In this process, carbonic anhydrase enzyme transports protons by $\mathrm{V}$-ATPase. Thus, the creation of $\mathrm{pH} 4.5$ in isolated area decomposes bone mineral components and exposes the organic material such as type I collagen, which can be decreased by CTSK (35).

\section{Cellular fusion in osteoclastogenesis}

The fusion of plasma membranes is a common phenomenon in almost every cell, such as the fusion of intracellular membranes which makes hormone secretion and neurotransmission possible. On the other hand, less is known about the mechanisms by which intercellular fusion occurs during osteoclastogenesis. Cell-cell fusion is a fundamental process in the generation of osteoclasts and multinucleated giant cells (36). The progenitor cells of osteoclast move to some specific location of hard tissues through the blood vessel, then these cells gradually form a 


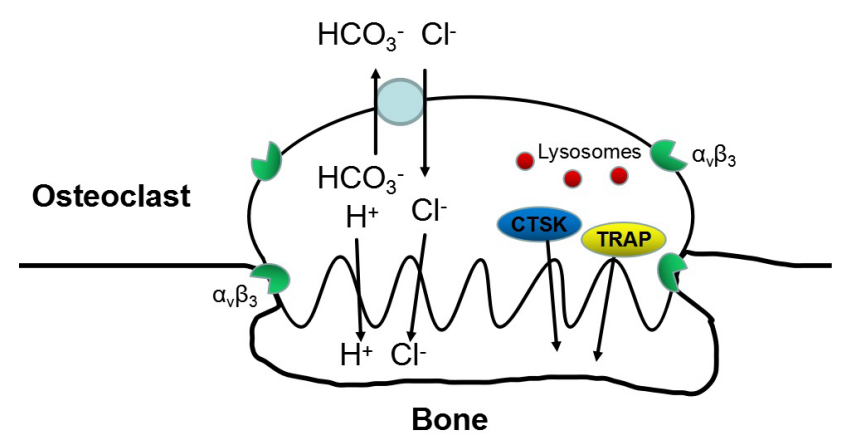

Figure 3. Mechanism of bone resorption. Osteoclasts release acid to the bone surface. Under acidic conditions, the inorganic minerals of bone are dissolved in the area of absorbing microenvironment. Osteoclasts degrade collagen by secreting several lysosomal enzymes, particularly CTSK and TRAP..

cluster and fuse with each other, and in the end become a mature osteoclast (37). Masaru put forward that cell fusion was the key process of osteoclast formation and regulating this process would provide potential therapeutic targets in bone diseases (38).

The cell fusion process in osteoclastogenesis involves many molecules, including dendritic cell-specific transmembrane protein (DC-STAMP) $(39,40)$, dendritic cell-specific protein, integrin, src family kinases, and integrin and metalloproteinase family proteins (41). Fusion regulatory protein FRP-1 is also in charge of cell fusion. The antibody of FRP-1 enhanced the generation of multinucleated giant cells and induced the generation of functional osteoclasts (42).

The process of cell fusion depends on DC-STAMP including the presence of phosphatidylserine. At the surface of the fusion area, molecules like Anx A5 and Syn-1 play an important role in forming protein scaffold structure to link the phosphatidylserine with S100A4 in cell surface to regulate the fusion process (43). On the other hand, the activation of $G$ protein-coupled receptor 119 can downregulate DC-STAMP to suppress preosteoclast fusion (44). Researchers find that actin binding LIM 1 (abLIM1) plays the role of negative control in cell fusion. Overexpression of abLIM1 suppresses osteoclast differentiation and formation. But when the expression of abLIM1 was downregulated by small interfering RNA, the formation and the marker genes of osteoclasts increased (45).

\section{Conclusion}

In summary, as a key cell in the bone metabolism system, studies on the mechanisms of osteoclast formation and activation have further deepened our understanding of osteoclasts. Research in bone immunology suggests that osteoclasts are involved in the regulation of the bone immune system, and the discovery of new signaling pathways, such as Semaphorin, may provide a new perspective on the mechanism of bone metabolic balance (46). With further research, especially in bone immunology $(47,48)$ and the RANK/RANKL pathway, it is expected to provide new ideas for the biological mechanism of osteoclasts and new directions for drug development for bone diseases.

\section{Acknowledgments}

The authors are grateful to YuJie Chang for proofreading this manuscript.

\section{Conflict of interest}

The authors declare that they have no conflict of interest.

\section{References}

1. Laperine O, Blin-Wakkach C, Guicheux J, Beck-Cormier S, Lesclous P. Dendritic-cell-derived osteoclasts: a new game changer in bone-resorption-associated diseases. Drug Discov Today. 2016;21(9):1345-54.

2. Han $Y$, You X, Xing W, Zhang Z, Zou W. Paracrine and endocrine actions of bone-the functions of secretory proteins from osteoblasts, osteocytes, and osteoclasts. Bone Res. 2018 May 24;6:16.

3. Yang D, Wan Y. Molecular determinants for the polarization of macrophage and osteoclast. Semin Immunopathol. 2019 Sep;41(5):551-563.

4. Kahn AJ, Simmons DJ. Investigation of cell lineage in bone using a chimaera of chick and quial embryonic tissue. Nature. 1975;258(5533):325-7.

5. Arai F, Miyamoto T, Ohneda O, Inada T, Sudo T, Brasel K, et al. Commitment and differentiation of osteoclast precursor cells by the sequential expression of c-Fms and receptor activator of nuclear factor kappaB (RANK) receptors. J Exp Med. 1999;190(12):1741-54.

6. Walker DG. Bone resorption restored in osteopetrotic mice by transplants of normal bone marrow and spleen cells. Science. 1975;190(4216):784-5.

7. Wu L, Luo Z, Liu Y, Jia L, Jiang Y, Du J, et al. Aspirin inhibits RANKL-induced osteoclast differentiation in dendritic cells by suppressing NF-kappaB and NFATc1 activation. Stem Cell Res Ther. 2019;10(1):375.

8. Tondravi MM, McKercher SR, Anderson K, Erdmann JM, Quiroz M, Maki R, et al. Osteopetrosis in mice lacking haematopoietic transcription factor PU.1. Nature. 1997;386(6620):81-4.

9. Udagawa N, Takahashi N, Akatsu T, Tanaka H, Sasaki T, Nishihara $\mathrm{T}$, et al. Origin of osteoclasts: mature monocytes and macrophages are capable of differentiating into osteoclasts under a suitable microenvironment prepared by bone marrow-derived stromal cells. Proc Natl Acad Sci USA. 1990 Sep;87(18):7260-4.

10. Monajemi M, Fisk S, Pang YCF, Leung J, Menzies SC, BenOthman R, et al. Malt1 deficient mice develop osteoporosis independent of osteoclast-intrinsic effects of Malt1 deficiency. J Leukoc Biol. 2019;106(4):863-77.

11. Mochizuki A, Takami M, Kawawa T, Suzumoto R, Sasaki $T$, Shiba $A$, et al. Identification and characterization of the precursors committed to osteoclasts induced by TNF-related activation-induced cytokine/receptor activator of NF-kappa B ligand. J Immunol. 2006 Oct 1;177(7):4360-8.

12. Grigoriadis $A E$, Wang ZQ, Cecchini MG, Hofstetter W, Felix $\mathrm{R}$, Fleisch HA, et al. c-Fos: a key regulator of osteoclastmacrophage lineage determination and bone remodeling. Science. 1994;266(5184):443-8.

13. Hanada R, Hanada T, Sigl V, Schramek D, Penninger JM. RANKL/RANK-beyond bones. J Mol Med (Berl). 2011 Jul;89(7):647-56.

14. Li J, Sarosi I, Yan XQ, Morony S, Capparelli C, Tan HL, et al. RANK is the intrinsic hematopoietic cell surface receptor that controls osteoclastogenesis and regulation of bone mass and calcium metabolism. Proc Natl Acad Sci USA. 2000 Feb 
15;97(4):1566-71

15. Narducci P, Nicolin V. Differentiation of activated monocytes into osteoclast-like cells on a hydroxyapatite substrate: an in vitro study. Ann Anat. 2009 Oct;191(4):349-55.

16. Iotsova V, Caamano J, Loy J, Yang Y, Lewin A, Bravo R. Osteopetrosis in mice lacking NF-kappaB1 and NFkappaB2. Nat Med. $1997 \mathrm{Nov;3(11):1285-9.}$

17. Simonet WS, Lacey DL, Dunstan CR, Kelley M, Chang MS, Luthy R, et al. Osteoprotegerin: a novel secreted protein involved in the regulation of bone density. Cell. 1997;89(2):309-19.

18. Dougall WC, Glaccum M, Charrier K, Rohrbach K, Brasel $\mathrm{K}$, De Smedt T, et al. RANK is essential for osteoclast and lymph node development. Genes Dev. 1999 Sep 15;13(18):2412-24.

19. Kong YY, Yoshida H, Sarosi I, Tan HL, Timms E, Capparell $\mathrm{C}$, et al. OPGL is a key regulator of osteoclastogenesis, lymphocyte development and lymph-node organogenesis. Nature. 1999;397(6717):315-23

20. Bucay N, Sarosi I, Dunstan CR, Morony S, Tarpley J, Capparelli C, et al. Osteoprotegerin-deficient mice develop early onset osteoporosis and arterial calcification. Genes Dev. 1998 May 1;12(9):1260-8.

21. Whyte MP, Wenkert $D$, McAlister WH, Novack DV, Nenninger $A R$, Zhang $X$, et al. Dysosteosclerosis presents as an "osteoclast-poor" form of osteopetrosis: comprehensive investigation of a 3-year-old girl and literature review. J Bone Miner Res. 2010 Nov;25(11):2527-39.

22. Lombardi MS, Gillieron C, Berkelaar M, Gabay C. Saltinducible kinases (SIK) inhibition reduces RANKL-induced osteoclastogenesis. PloS one. 2017;12(10):e0185426.

23. Taub M. Salt Inducible kinase signaling networks: implications for acute kidney injury and therapeutic potential. Int J Mol Sci. 2019 Jun 30;20(13):3219.

24. Uehara S, Udagawa N, Kobayashi Y. Regulation of osteoclast function via Rho-Pkn3-c-Src pathways. J Oral Biosci. 2019;61(3):135-40.

25. Weivoda MM, Ruan M, Hachfeld CM, Pederson L, Howe A, Davey RA, et al. Wnt signaling inhibits osteoclast differentiation by activating canonical and noncanonical cAMP/PKA pathways. J Bone Miner Res. 2019 Aug;34(8):1546-1548.

26. Yin $Y$, Ding $L$, Hou $Y$, Jiang $H$, Zhang J, Dai $Z$, et al Upregulating MicroRNA-410 or Downregulating wnt-11 increases osteoblasts and reduces osteoclasts to alleviate osteonecrosis of the femoral head. Nanoscale Res Lett. 2019 Dec 18;14(1):383.

27. Ashley JW, Ahn J, Hankenson KD. Notch signaling promotes osteoclast maturation and resorptive activity. J Cell Biochem. 2015 Nov;116(11):2598-609.

28. Teitelbaum SL. The osteoclast and its unique cytoskeleton. Ann N Y Acad Sci. 2011 Dec;1240:14-7.

29. McHugh KP, Hodivala-Dilke K, Zheng MH, Namba N, Lam J, Novack D, et al. Mice lacking beta3 integrins are osteosclerotic because of dysfunctional osteoclasts. J Clin Invest. $2000 \mathrm{Feb}$;105(4):433-40.

30. Minkin C. Bone acid phosphatase: tartrate-resistant acid phosphatase as a marker of osteoclast function. Calcif Tissue Int. 1982;34(3):285-90.

31. Motyckova G, Weilbaecher KN, Horstmann M, Rieman DJ, Fisher DZ, Fisher DE. Linking osteopetrosis and pycnodysostosis: regulation of cathepsin $\mathrm{K}$ expression by the microphthalmia transcription factor family. Proc Natl
Acad Sci USA. 2001 May 8;98(10):5798-803.

32. Ahn SH, Chen Z, Lee J, Lee SW, Min SH, Kim ND, et al. Inhibitory effects of 2N1HIA (2-(3-(2-Fluoro4-Methoxyphenyl)-6-Oxo-1(6H)-Pyridazinyl)-N-1 HIndol-5-Ylacetamid e) on osteoclast differentiation via suppressing cathepsin K expression. Molecules. $2018 \mathrm{Nov}$ 29;23(12):3139.

33. Soe K, Delaisse JM. Time-lapse reveals that osteoclasts can move across the bone surface while resorbing. J Cell Sci. 2017 Jun 15;130(12):2026-2035.

34. Soysa NS, Alles N. Osteoclast function and bone-resorbing activity: An overview. Biochem Biophys Res Commun. 2016;476(3):115-20.

35. Calle $\mathrm{Y}$, Jones GE, Jagger C, Fuller K, Blundell MP, Chow $\mathrm{J}$, et al. WASp deficiency in mice results in failure to form osteoclast sealing zones and defects in bone resorption. Blood. 2004;103(9):3552-61.

36. Pereira M, Petretto E, Gordon S, Bassett JHD, Williams GR, Behmoaras J. Common signalling pathways in macrophage and osteoclast multinucleation. J Cell Sci. 2018 Jun 5;131(11):jcs216267.

37. Teitelbaum SL. Bone resorption by osteoclasts. Science. 2000;289(5484):1504-8.

38. Ishii M, Saeki Y. Osteoclast cell fusion: mechanisms and molecules. Mod Rheumatol. 2008;18(3):220-7.

39. Mbalaviele G, Chen H, Boyce BF, Mundy GR, Yoneda T. The role of cadherin in the generation of multinucleated osteoclasts from mononuclear precursors in murine marrow. J Clin Invest. 1995 Jun;95(6):2757-65.

40. Yagi M, Miyamoto T, Sawatani $\mathrm{Y}$, Iwamoto K, Hosogane N, Fujita N, et al. DC-STAMP is essential for cell-cell fusion in osteoclasts and foreign body giant cells. J Exp Med. 2005;202(3):345-51.

41. Verrier S, Hogan A, McKie N, Horton M. ADAM gene expression and regulation during human osteoclast formation. Bone. 2004;35(1):34-46

42. Higuchi S, Tabata N, Tajima M, Ito M, Tsurudome M, Sudo A, et al. Induction of human osteoclast-like cells by treatment of blood monocytes with anti-fusion regulatory protein-1/ CD98 monoclonal antibodies. J Bone Miner Res. 1998 Jan;13(1):44-9.

43. Verma SK, Leikina E, Melikov K, Gebert C, Kram V, Young MF, et al. Cell-surface phosphatidylserine regulates osteoclast precursor fusion. J Biol Chem. 2018 Jan 5;293(1):254-270.

44. Kim HJ, Yoon HJ, Park JW, Che X, Jin X, Choi JY. G protein-coupled receptor 119 is involved in RANKL-induced osteoclast differentiation and fusion. J Cell Physiol. 2019 Jul;234(7):11490-11499.

45. Narahara H, Sakai E, Yamaguchi $Y$, Narahara S, Iwatake M, Okamoto K, et al. Actin binding LIM 1 (abLIM1) negatively controls osteoclastogenesis by regulating cell migration and fusion. J Cell Physiol. 2018 Jan;234(1):486-499.

46. Fukuda T, Takeda S, Xu R, Ochi H, Sunamura S, Sato T, et al. Sema3A regulates bone-mass accrual through sensory innervations. Nature. 2013;497(7450):490-3.

47. Foger-Samwald U, Dovjak P, Azizi-Semrad U, KerschanSchindl K, Pietschmann P. Osteoporosis: pathophysiology and therapeutic options. EXCLI J. 2020;19:1017-37.

48. Hsu E, Pacifici R. From osteoimmunology to osteomicrobiology: how the microbiota and the immune system regulate bone. Calcif Tissue Int. 2018;102(5):512-21. 